\title{
Use of computer aided drafting for analysis and control of posture in manual work
}

\author{
S.S. Ulin*, T.J. Armstrong* and R.G. Radwin ${ }^{\dagger}$ \\ "Center for Ergonomics, The University of Michigan, Ann Arbor, MI 48109-2117, USA \\ ${ }^{\dagger}$ Department of Industrial Engineering, University of Wisconsin-Madison, Madison, WI 53706-1512, USA
}

Computer aided design (CAD) in conjunction with digitised anthropometric manikins can be used for analysis and control of stressful work postures, one of the most frequently cited occupational risk factors of upper extremity cumulative trauma disorders. This paper describes the use of macros for manipulating manikins and workstation components and for designing the workplace. AutoCAD, a popular computer aided design software package, was used to demonstrate the feasibility of these concepts. Specifically, macros are used for drawing work equipment using parametric designs, manipulating manikins and analysing jobs. In comparing the macros to the use of primitive CAD commands, the macros not only decrease the amount of time needed to create workstation components, but they also make the task easier for the user and decrease the risk of errors. Despite the limitation of anthropometric data and manikins, CAD is an effective method for identifying postural stresses and redesigning the workstation to control the identified stresses. Keywords: Computer aided design, posture, anthropometric characteristics, manual work, occupational hazards, manikins,
workplace design

\section{Introduction}

This paper is concerned with identifying stressful postures and redesigning workstations and work equipment to eliminate these stresses. The National Institute of Occupational Safety and Health (NIOSH) investigators have found incidence rates for cumulative trauma disorders at specific US worksites range between $3 \%$ and $58 \%$ of the employees (Putz-Anderson, 1988; Silverstein, 1985; Armstrong et al, 1982). The reported risk factors for occupational trauma disorders include repetitive exertions (Hagberg, 1982), awkward postures (Herberts et al, 1981; Hoffmann, 1981; Tichauer, 1976; Armstrong and Chaffin, 1979), mechanical stress concentrations on the hand and arm (Armstrong, 1983; Tichauer, 1976), vibration (Armstrong et al, 1987), and cold (Williamson et al, 1984), although this paper is primarily concerned with identifying and eliminating stressful upper extremity postures.

Work situations resulting in physical contact with the workstation or the potential for working in awkward postures can sometimes be identified by positioning a drawing board manikin over a sketch of the workstation. Through a series of designs developed by trial and error the workplace can be designed to minimise these awkward postures. Although drawing board manikins are a useful design tool, they have several serious limitations: (1) they are only available in limited percentiles and scales, (2) they are not easily adapted for tasks which do not lie in the sagittal plane of the body, and (3) they are awkward to manipulate. Armstrong et al
(1986) have shown that some of these limitations can be overcome by using digitised manikins with computer aided drafting (CAD).

This paper describes using computer aided design and a set of digitised manikins for analysis and control of stressful postures. Programming enhancements to facilitate manikin manipulation, manikin adjustments for reaching outside of the sagittal plane, and examples of workstation designs to control work postures are discussed. In addition, this paper describes the use of macros for manipulating the manikins and workstation components, and for assisting in the analysis of the resulting design.

\section{Computer aided design}

CAD can be used to design a variety of objects. For example, engineers can use CAD to design consumer goods, vehicles and what is dealt with in this paper, manufacturing workstations. At its simplest level, CAD is used like a set of drafting instruments for constructing lines and shapes on a computer display. This is accomplished through commands for drawing primitive shapes that are provided in the software package. The user determines the placement of those lines the same way as in manual drawings. The drawings may be plotted or stored on electronic media for later editing and analysis.

At a higher level, CAD software can be programmed to determine where to place lines and shapes. This programming may be accomplished within the CAD environment using 
specialised programming languages or standard languages such as FORTRAN, PASCAL, C, LISP, etc. The primitive drawing commands can be combined with a programming language for building macros. Table 1 lists a few commercially available CAD software packages and the programming language each one utilises.

At the highest level, $\mathrm{CAD}$ provides the integration of graphics and a knowledge base into an expert support system to help identify and control stressful work postures. An expert support system helps a trained user interpret and analyse data, so that a difficult problem can be effectively solved (Luconi et al, 1986). In contrast, expert systems which have grown out of the computer science field of artificial intelligence are computer programs that utilise expert know. ledge and symbolic reasoning to solve correctly difficult problems in a narrow domain. For a well-trained user in a specific problem domain, it is not necessary to use an expert system.

CAD systems acquire much greater flexibility through the use of macros because the user can draw and manipulate objects on the screen using only a few inputs as opposed to inputting two or three times as many primitive commands to accomplish the same result. Macros for generating specific objects, for instance, need only the essential parameters, such as location and orientation. A macro that generates a specific component of the workstation can be structured so the only inputs necessary are the parameters describing the object. Consequently, a macro provides customised workstation components and drawings for the user's specific requirements. This saves time since the user does not have to produce an entire drawing using basic primitives only because a few of the dimensions of an object have been changed. Macros may also be used for calculating the exact location and orientation of objects. This is usually more accurate than visually positioning parts. The development and use of macros will be illustrated below.

It is also possible to enhance CAD software packages by writing programs using conventional programming languages and passing that information through ASCII files to the CAD system. Information contained in database files can also be sent to a CAD program for generating objects within the CAD environment.

AutoCAD, a popular computer aided design software package, was selected as the environment for use in developing a system to guide the user in workstation design. AutoCAD was chosen for several reasons. First, this software runs on micro-computers such as the IBM-PC/PS2, a computer often used by engineers in industry. Second, the low price of the desktop personal computer makes it more economically feasible than a $C A D$ package that requires a mainframe

Table 1: Examples of CAD software packages and the language they use for building macros

\begin{tabular}{ll}
\hline Software & Language \\
\hline AUTOCAD & AUTOLISP \\
CADKEY & CADL \\
VERSACAD & CPL \\
AUTO-TROL & EAGLE \\
PERSONAL DESIGNER'S & UPL \\
\hline
\end{tabular}

computer. Third, AutoCAD includes AutoLISP, a LISP type interpreter which combines drawing commands with LISP code, and can be used for writing programs to manipulate and analyse drawings. The workstation analysis routines, which can be written using Auto LISP in conjunction with the graphics environment of AutoCAD, provide a powerful tool for the workplace/tool designer

\section{Applying CAD to workstation design}

To demonstrate how AutoCAD could be customised to facilitate the workstation design process, first, two-dimensional anthropometric manikins were digitised. Next, AutoLISP routines for parametric designs of work equipment and for manipulating manikins were written. Finally, a few AutoLISP procedures were written to perform specific job analysis routines.

To demonstrate the utility of the Auto LISP macros, drawings were constructed and edited using both primitive commands and the macros. These drawings contain examples of building a workbench, constructing a stocktable, assembling a manikin, configuring the manikin's arms to reach to a specified location, and analysing the manikin's posture

\section{Anthropometric manikins}

$\mathrm{CAD}$ in combination with digitised two-dimensional manikins can be a useful beginning when designing manufacturing workstations. Other sources of anthropometric information which can be used in the design stage include tabular data, two-dimensional drawing board manikins, three-dimensional computer man-models, and test subjects interacting with a prototype.

CAD systems with three-dimensional capabilities have been developed and the capabilities of these systems generally include: modelling man in three dimensions; allowing for clothing and other personal equipment; and demonstrating the interactions between workstations or products and the operators early in the design process (Rothwell and Hickey, 1986). The SAMMIE system (Case et al, 1986) was intended to enhance the quality of designs by providing a variety of three-dimensional man-models which can easily interact with the current workstation or product design and by allowing evaluation of that interaction. Two other systems - COMBIMAN (Evans, 1982) and TEMPUS (Badler et al, 1985) with three-dimensional man-models - were originally developed to evaluate interactions in aircrew stations. CAD systems which included three-dimensional man-models offer opportunities for the designer to analyse human involvement with the workstation or product they are planning.

Manikins developed by the US Air Force which closely approximate 5 th and 95 th percentile females and males (Kennedy, 1982) were selected for use in designing workstations because they are based on up-to-date data and are well-documented. These manikins were digitised as three segments for each of the extremities, one for the torso and one for the head and neck (Armstrong et al, 1986). Presently, a user can view how the manikins interact with a new workstation design. Ideally, to help solve design problems, the system should be able to guide the user to preferred work postures and tool/workstation configurations.

AutoLISP routines for parametric deaigns of work equipment

Macros can be written within a CAD environment to generate various components of the workstation. The manikins 
interact with the equipment and tools contained in the workstation under design. This will allow a new workstation to be tested while it is still 'on the drawing board'. Consequently, several macros for generating generic types of workstations and hand tools have been developed. Parametric designs are used to construct drawing of various workstation components so a user can easily customise and change the objects contained in a specific workstation drawing.

\section{Example 1: Creating a workbench}

The first example is a macro to generate a workbench. Using primitive commands, a workbench can be generated through a series of eight LINE commands. The user must calculate the $x$ and $y$ co-ordinates for each point of the legs and table top based on the desired dimensions for the work. bench. For example, for a flat workbench with height, $h$, length, $l$, and depth of table top and legs, $d$, the points are calculated as follows:

$$
\begin{aligned}
& x_{0}, y_{0} \\
& x_{0}, y_{0}+h \\
& x_{0}+l, y_{0}+h \\
& x_{0}+l, y_{0} \\
& x_{0}+l-d, y_{0} \\
& x_{0}+l-d, y_{0} \\
& x_{0}+l-d, y_{0}+h-d \\
& x_{0}+d, y_{0}+h-d \\
& x_{0}+d, y_{0}
\end{aligned}
$$

This is illustrated in Fig. $1 \mathrm{~A}$ for a $91 \mathrm{~cm}$ high and $76 \mathrm{~cm}$ wide workbench $(h=91, l=76, d=7)$ which is located $50 \mathrm{~cm}$ from the lower left corner of the screen $\left(x_{0}=50, y_{0}=0\right)$. The workbench is a very simple example, but the complexity increases rapidly as the number of lines increase or oblique lines and curves are added.

A macro for drawing a workbench requiring an insertion point, height and length of the workbench, depth of the top, width of the legs, and angle of the table top as input variables can be written in AutoLISP. The use of this macro is illustrated in Figs. 1B and 1C. The AutoLISP code which constructs a workbench is listed here with descriptive comments in brackets.

\section{AutoLISP code for workbench}

(defun workbench 0

\section{(Retrieve input parameters from the user)}

(setq P1 (Getpoint "InEnter the insertion point of the workbench (left-most point): "))

(setq H (Getreal "InEnter the height of the table: "))

(setq L (Getreal "InEnter the length of the table: "))

(setq D (Getreal "InEnter the depth of the table top: "))

(setq W (Getreal "InEnter the width of the legs: "))

(setq alpha (Getreal "InEnter the angle of the table top: "))

(Compute the height which will be added to the right leg because of a slanted table top)

$(\operatorname{setq} b(* L(\sin (* 0.0175$ alpha $))))$

\section{(Insert left leg)}

(Insert a $1 x 1$ square at the insertion point with width $(x)$, $W$, and height (y), $H-D$, and no rotation)

(command "INSERT" "SQUARE" P1 W (-HD) "”)

\section{(Insert right leg)}

(Insert a $1 x I$ square $L$ units to the right of the insertion point with width $(x),-W$, height $(y)$, $(H+b)-D$, and no rotation)

(command "INSERT" "SQUARE" (list (+ L (car P1)) (cadr P1)) (-W) (-(+Hb) D) "'")

(Insert workbench top)

(Insert a $1 x 1$ square at the top left corner of the left table leg with width $(x), L$, height (y), $-D$, and angle of rotation, alpha)

(command "INSERT" "SQUARE" (list (car P1) (+H (cadr P1))) L(-D) alpha)

(End of workbench macro)

This macro can be invoked by calling it directly, or by making it part of a menu selection. Other programming

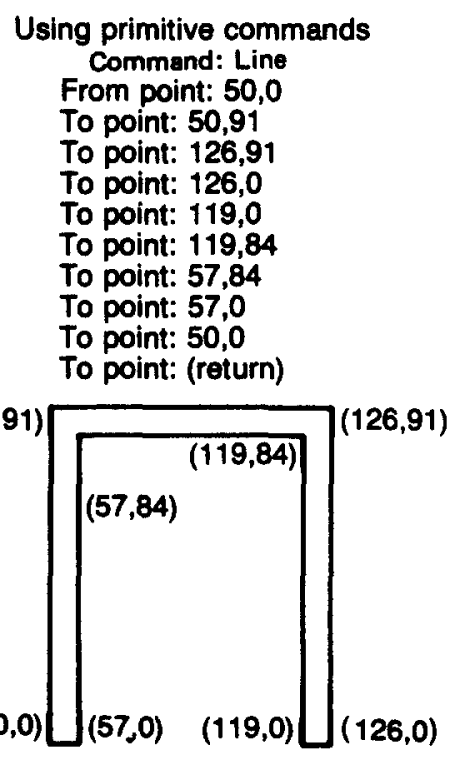

a
Insertion point of workbench: $\mathbf{5 0 , 0}$ Height of workbench: $91 \mathrm{~cm}$ Length of workbench: $76 \mathrm{~cm}$ Depth of table top: $7 \mathrm{~cm}$ Width of legs: $7 \mathrm{~cm}$ Angle of table top: 0

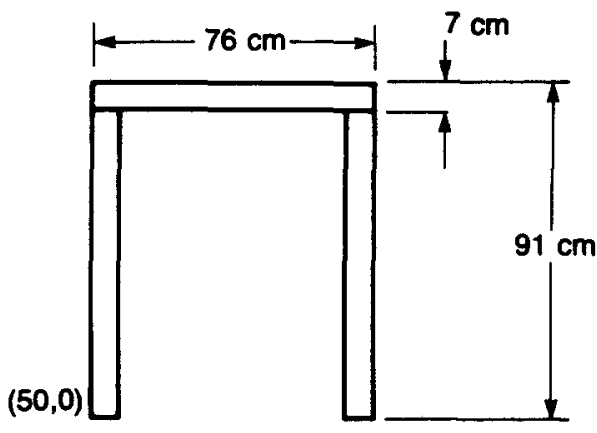

b
Using a macro

Insertion point of workbench: 50,0 Height of workbench: $91 \mathrm{~cm}$ Length of workbench: $76 \mathrm{~cm}$ Depth of table top: $7 \mathrm{~cm}$ Width of legs: $7 \mathrm{~cm}$ Angle of table top: $15 \mathrm{~cm}$

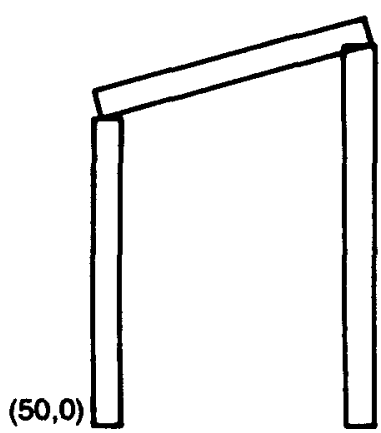

$(50,0)$

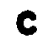

Fig. 1 (a) Creating a workbench with primitive commands; (b) creating a workbench using a macro; (c) creating a workbench with a slanted table top using a macro 
languages such as Pascal or dBASE can be used to construct the same workbench.

As additional specifications and commands are added to the macro, the advantage with respect to session time over using primitive commands may be lost, but advantages in ease of use and risk of errors are gained, since the conceptual load of the user is minimised (Rubinstein et al, 1984). For example, it is easier for a user to specify the thickness of the top of the workbench than to calculate and enter all of the necessary $x$ and $y$ co-ordinates. The advantage of these routines becomes particularly evident when manipulating manikins.

\section{Example 2: Stock table}

The STOCKTABLE macro allows a user to construct a stock table, pallet and a representation of the stock on top of the pallet. The insertion point of the stock table, the length and height of the stock table, the height of the pallet, and the length and height of the stock are entered. Next, the system draws the stock table, pallet and a representation of the stock based on the user's inputs. This routine is illustrated in Fig. 2 . The STOCKTABLE macro requires six inputs and no user computations. To draw a stock table using primitive commands, however, would require 13 inputs and each of those inputs includes an $x, y$ co-ordinate pair which the user must compute based on the dimensions of the stock table. An error in any of these computations would require erasing and re-computing all successive points. Without a macro, this could become a very time-consuming process.

\section{AutoLISP routines for manipulating body postures}

Manikin body segments were digitised and saved as blocks so that they can be inserted as needed into any workstation drawing. To facilitate the use of the manikins, macros were

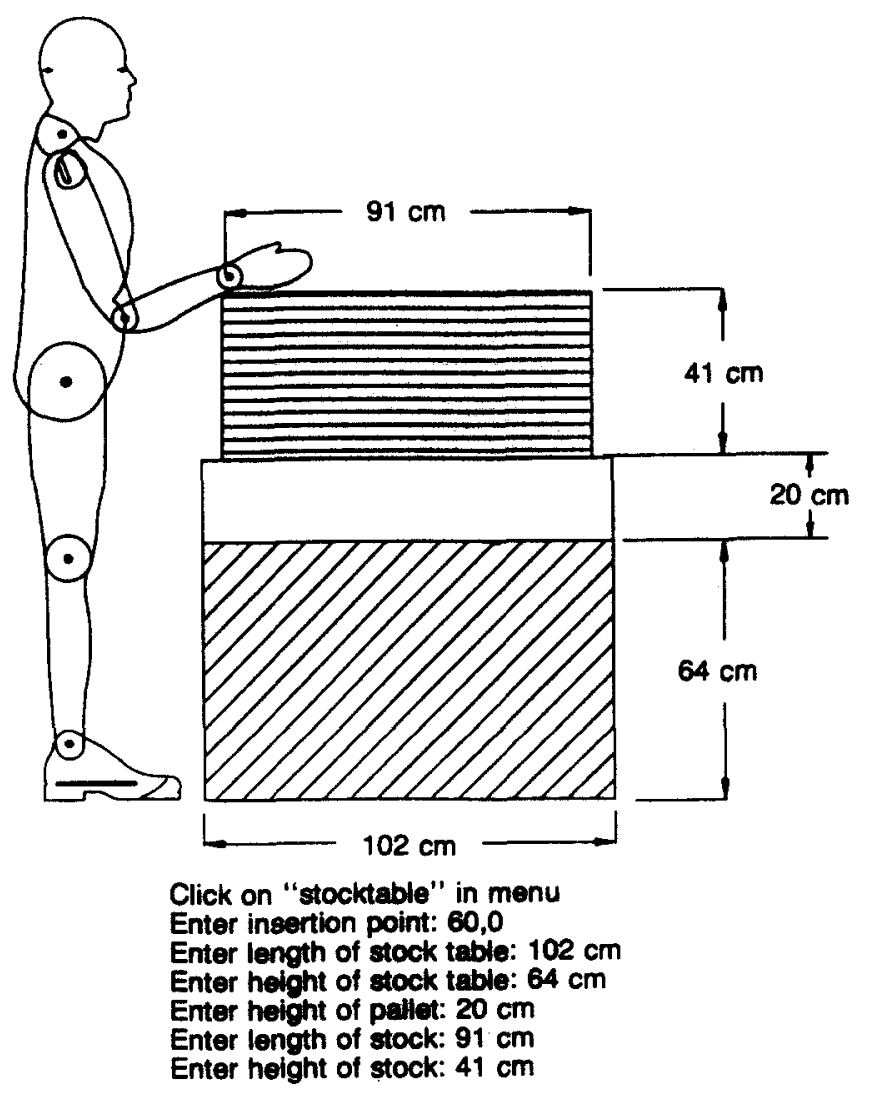

Fig. 2 Constructing a stock table written to generate the manikin based on the specified insertion point for the foot. A mouse is used to rotate the various body segments into the desired posture as they appear on the computer screen. It is also possible to enter a numeric value for specifying a rotation angle for each body segment. This routine eliminates the need to line up insertion points of the various body segments visually, and provides the data for the computer to calculate the insertion point for the next body link based on the position of the previous segments.

\section{Example 3: Assembling a manikin}

Fig. 3 illustrates two methods for assembling a specific manikin, using both primitive commands and a macro. Without the macro, the user would have to insert each body link and specify its insertion point, $x$-scale factor, $y$-scale factor, and angle of rotation (Fig. 3A). The user must specify 48 pieces of input information to insert a manikin using primitive commands and the insertion point for each body segment must be visually indicated (or manually calculated). Using the macro greatly increases the ease and accuracy with which a manikin can be inserted into a drawing (Fig. 3B) and reduces the number of input variables to eight. Still more reduction in the number of required inputs could be achieved if a set of common postures were defined. Then, the user could merely choose a beginning posture configuration for the manikin.

After the manikin is inserted into a drawing, the designer can alter the workstation and test various postures to investigate the interaction between the worker, workstation and tools. Primitive commands for moving or rotating previously inserted blocks can be time consuming because the movement of one body segment usually requires moving other body segments. Consequently, macros were written for rotating one body segment at a time, about the inferior body segment. After the user has indicated the new position of a body segment linkage, the superior body segments are moved based on the change of the first body segment, while maintaining their original orientation.

\section{Example 4: Reach}

The primitive commands required for relocating the manikin's upper limbs are shown in Fig. 4. Fig. 4A shows the initial posture of the manikin and the box which he must reach, and Fig. 4B shows the primitive commands which would be used to re-configure the manikin's arms for reaching the box. In reality, several iterations may be required to obtain even an approximation of the desired posture, thereby greatly increasing the number of primitive commands used to position the upper limbs. One iteration of re-configuring the manikin's upper limbs consists of 15 pieces of input information. $A$ REACH macro can be used for repositioning the upper limbs which requires only one input, the $x$ and $y$ co-ordinates of the new hand location.

The REACH macro determines the corresponding positions of the upper arm, lower arm and hand for a specific point the manikin must reach. The user specifies the shoulder location (or it may be previously known) and the new hand location. The system first determines if it is possible to reach the new hand location (see Fig. 4C). If it is possible to reach the new hand location, the upper limbs are repositioned. Since it is desirable to keep the wrist in a neutral posture (Armstrong and Silverstein, 1987), the system defines the wrist angle to be the same angle as the forearm to minimise wrist deviation. If necessary, the wrist posture may be specified based on a handle design. 


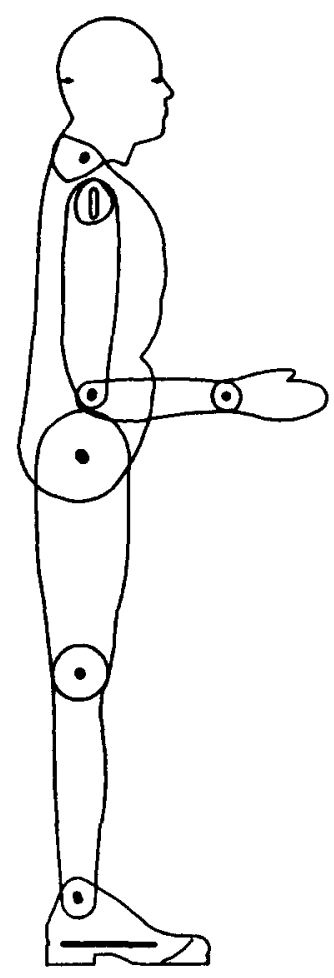

Primitive commands

Command: Insert Blockname: Foot Insertion point: 16,0

Rotation angle: 0

$X$-scale factor: 1

Y-scale factor: 1

Command: Insert Blockname: Lleg Insertion point: $19,5.6$

Rotation angle: 90

$X$-scale factor: 1

Y-scale factor: 1

Command: Insert Blockname: Uleg Insertion point: 19, 23.2

Rotation angle: 90

$X$-scale factor 1

Y-scale factor: 1
Command: Insert

Blockname: Torso Insertion point: $19,40.8$

Rotation angle: 90

$X$-scale factor: 1

Y-scale factor: 1

Command: Insert Blockname: uarm Insertion point: 19.7,61.2

Rotation angle: 270

$X$-scale factor:

Y-scale factor: 1

Command: Insert Blockname: Hand Insertion point: $30.7,45.5$

Rotation angle: 0

$X$-scale factor: 1

$Y$-scale factor: 1

Command: Insert Blockname: hdnk Insertion point: $19,64.9$

Rotation angle: 90

$X$-scale factor: 1

Y-scale factor: 1

Fig. 3A Building a manikin with primitive commands

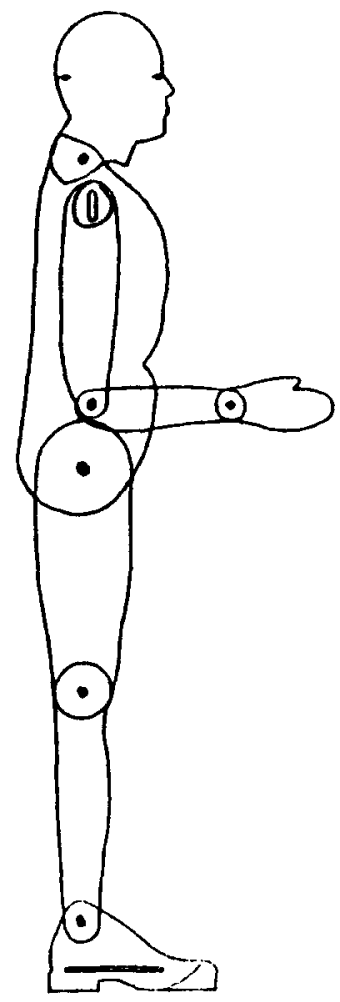

Using a MACRO:

Enter the foot localion: 16,0 Indicate rotation angle of lower leg Indicate rotation angle of upper leg Indicate rotation angle of torso indicate rotation angle of upper arm Indicate rotation angle of lower arm indicate rotation angle of hand Indicate rotation angle of head/neck

Fig. 3B Building a manikin using a macro

Command: Insert

Blockname: Larm

Insertion point: $19.7,45.5$

Rotation angle: 0

$X$-scale factor 1

Y-scale factor: 1

\section{AutoLISP routines for job analysis}

\section{Example 5: Posture analysis}

A POSTURE analysis toutine was written for identifying the angles of the wrist, lower arm, upper arm and torso with respect to a common reference point. The user first positions the manikin at the workstation. The program then compares each joint position with a desired joint posture. If any of the body segments are not in a neutral posture, the system writes a message to the user that limb $X$ is in mild (or severe) flexion, extension, radial deviation or ulnar deviation (Keyserling, 1986; Armstrong and Chaffin, 1979; Armstrong et al, 1984; Tichauer, 1966, 1976; Hoffman, 1981). The user may utilise this information (i e, minimise upper arm flexion) for designing or redesigning a workstation. It will be very easy to update the criterion used in this macro as new posture data become,available.

\section{Sample workstations redesigned using CAD}

It is the combination of the CAD system, the digitised manikins, and the AutoLISP macros that make this type of approach so useful for the user who is designing or redesigning manual workstations. In particular, this approach helps significantly in five ways during the process of job design:

1. Determine how the workstation design affects workers with varied anthropometry (i e, 95 th percentile male and 5 th percentile female stature).

2. Identify stressful postures (Keyserling, 1986; Armstrong and Chaffin, 1979; Armstrong et al, 1984; Tichauer, 1966, 1976; Hoffman, 1981).

3. Alter workstation designs until identified stresses are minimised.

4. Specify the workstation requirements.

5. Provide pictorial evidence of how workstations can be improved to management during the 'paper' stage of design development. 


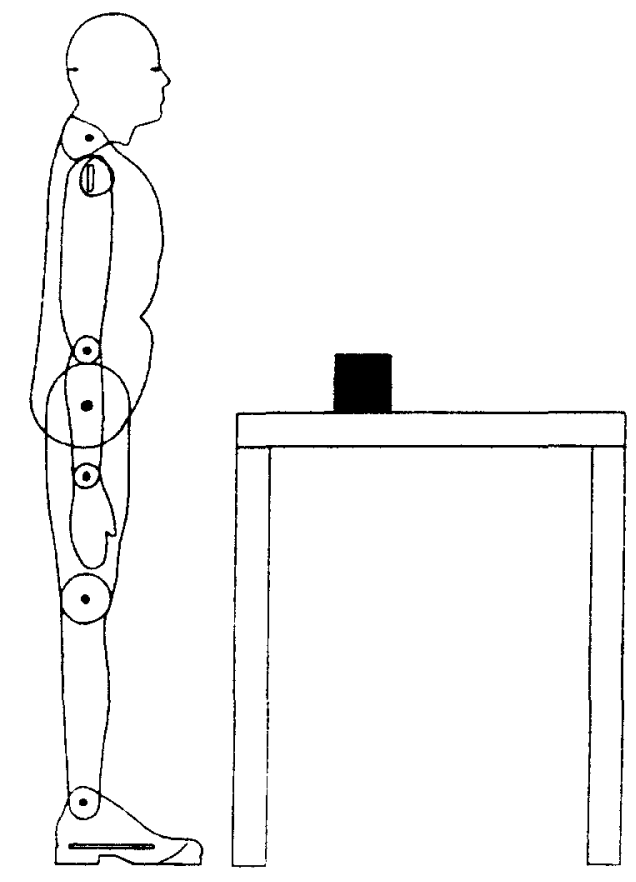

Fig. 4A Initial manikin posture

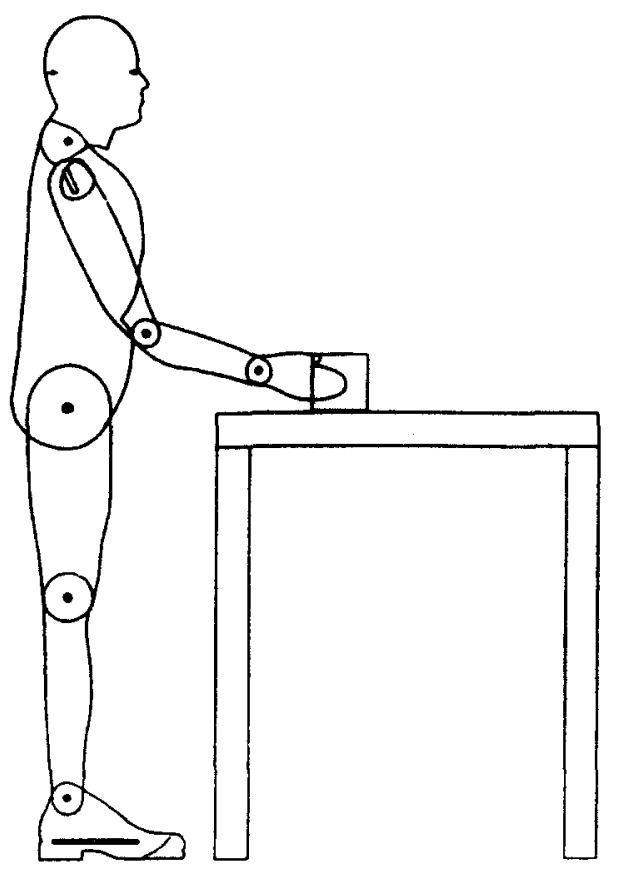

Using primitive commands

Command:Change

Select objects: (elect upper arm)

PropertieakChange point >: (roturn)

Enter block insertion point: (retum)

Enter rotation angle: (indicate arm rotation)

Command:Change

Select objects: (select lower arm)

Enter block ineortion point: (click on point)

Enter rotation angle: (indicete arm rotation

Command: Change

Select objects: (select hand)

Propertiea/<Change point>: (return)

Enter block insertion point: (click on point)

Enter rotation angle: (indicale hand rotation)

Fig. 4B Configuring a manikin to reach to a specified point using primitive commands

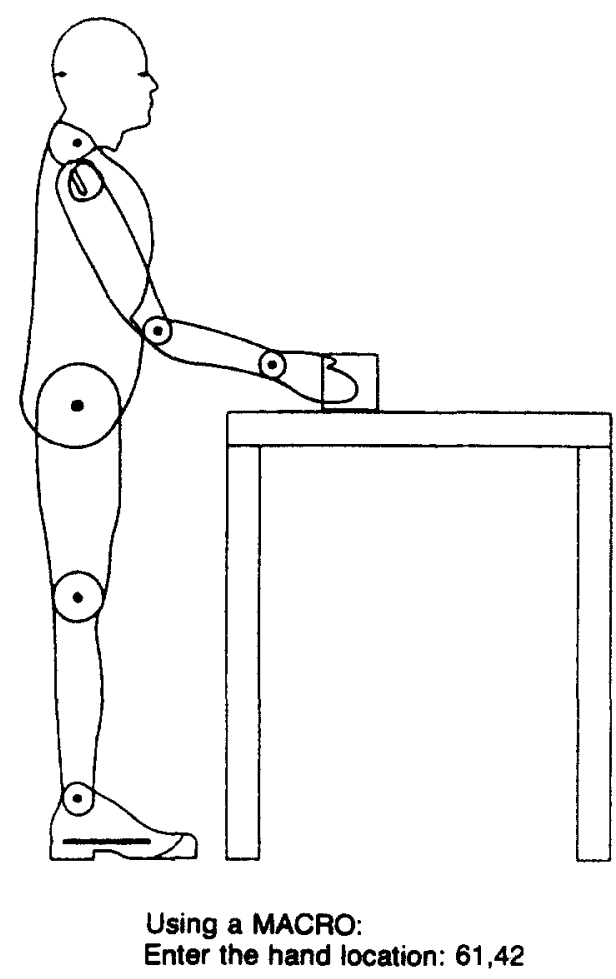

Fig. 4C Configuring a manikin to reach to a specified point using the reach macro

In the next two examples the usefulness of using CAD for redesigning workstations is shown.

Fig. 5 depicts a worker lifting a metal blank off the top of a stack. Workstation drawings including both a large male and a small female were drawn to demonstrate the effect of workstation design for a population with varied anthropometries (Kennedy, 1982; Armstrong et al, 1986). The manikins were chosen to represent the small and large size extremities in the working population. Therefore, if a job is designed so that both extremes are accommodated, it can be generalised that the design will accommodate most of the working population. In this example, the stock table and pallet are a fixed height, but the height of the stock ranges from 0 to $41 \mathrm{~cm}$ high. Picking up a blank from the top of the stack requires deviation of the worker's wrist. The upper arm of the large male is elevated, while the upper arm of the small female is in extreme flexion. The small female must reach far above shoulder height to pick up a blank from the top of the stack.

The postural problems identified in Fig. 5 may be eliminated by putting the stack of blanks on an adjustable lift table. Fig. 6 shows that when the top of the stack is kept at or just below elbow height, the wrist deviation is eliminated and so is the shoulder flexion. In this example, a CAD system was used to identify the postural stresses of this operation and determine the parameters for a solution to correct these identified problems.

The series of drawings in Figs. 7A and 7B demonstrate how a large male and a small female interact with conveyors which range in height between 81 and $119 \mathrm{~cm}$. If the objective is to minimise the torso and upper arm flexion, and the workstation constraints include positioning the conveyor at a fixed height, then it becomes apparent from the series 


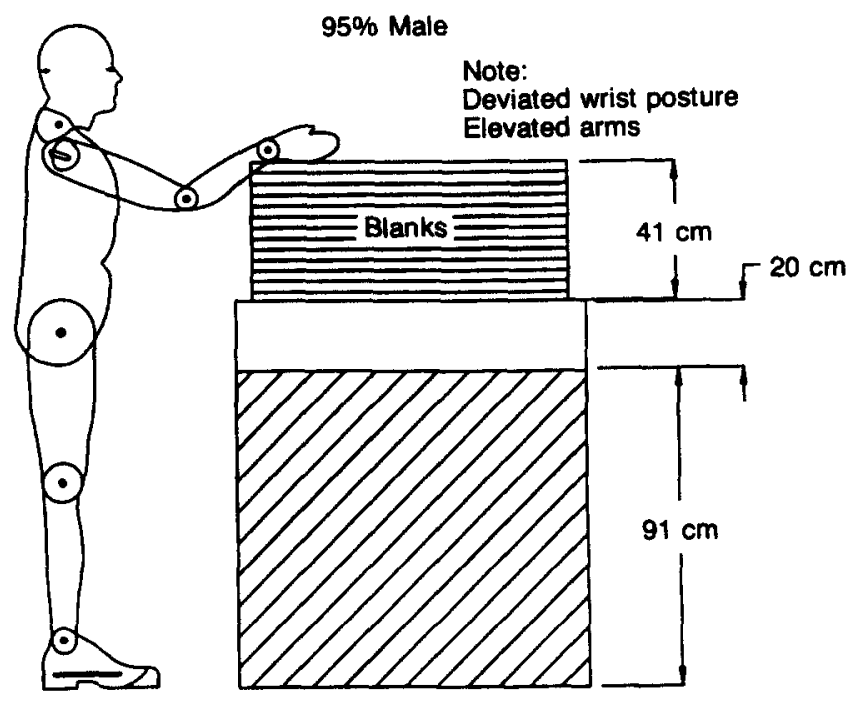

$5 \%$ Female

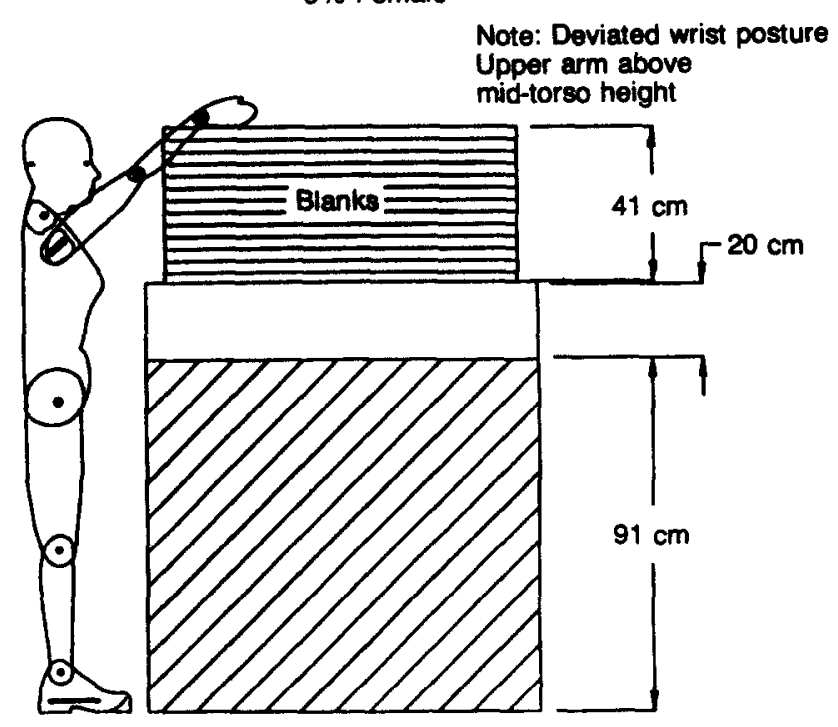

Fig. 5 Blanks on a stationary stock table

of drawings at which height the conveyor should be located. A conveyor height of $96 \mathrm{~cm}$ can minimise the postural stresses for both the large male and the small female.

\section{Limitations of anthropometric data and manikins}

At the present time, manikins are a very worthwhile tool for designers when used for determining the dimensions of a workstation, although anthropometric data have led people to believe in the 'mythical person'. The mythical person (or manikin) is someone whose body segments do not have substantial variation. This person is considered to be mythical because a given population of operators contains individuals having considerable variation in the relative sizes of their body segments. For example, an individual can be 95 th percentile in stature and only 75 th percentile in elbow height or upper arm length (Roebuck et al, 1975). Consequently, when designing a workstation, the designer should use manikins of both the small and large percentile individuals to account for a wide population range in the design (Roebuck et al, 1975; Kennedy, 1982). As new anthropometric data become available, the body segments of the manikins can be easily updated to reflect the new data.
Designing workstations to accommodate the working population is difficult because of the large variability in people. This is attributed to three factors (NASA, 1978):

(1) Intra-individual variability accounts for size changes that occur during an individual's adult life because of ageing, nutrition or the environment.

(2) Inter-individual variability refers to the distinction between the sexes and people from different ethnic origins or various geographical locations.

(3) Secular variability refers to the variability between different generations.

It is apparent that the working population, particularly in the US, is extremely variable.

To account for this large variability in population, Bittner et al (1987) developed a set, or family, of manikin dimensions. Their research culminated in a 17 -member cadre of manikins. The visual and reach accommodation of the manikins was compared with the same measurements from a 400 -member random sample of individuals. The comparison showed that the manikins accounted for $98.25 \%$ of the visual and reach accommodation of the subjects. It is important to note that whether a designer is using the 17 -member cadre of manikins or representations of the large and small percentiles of the
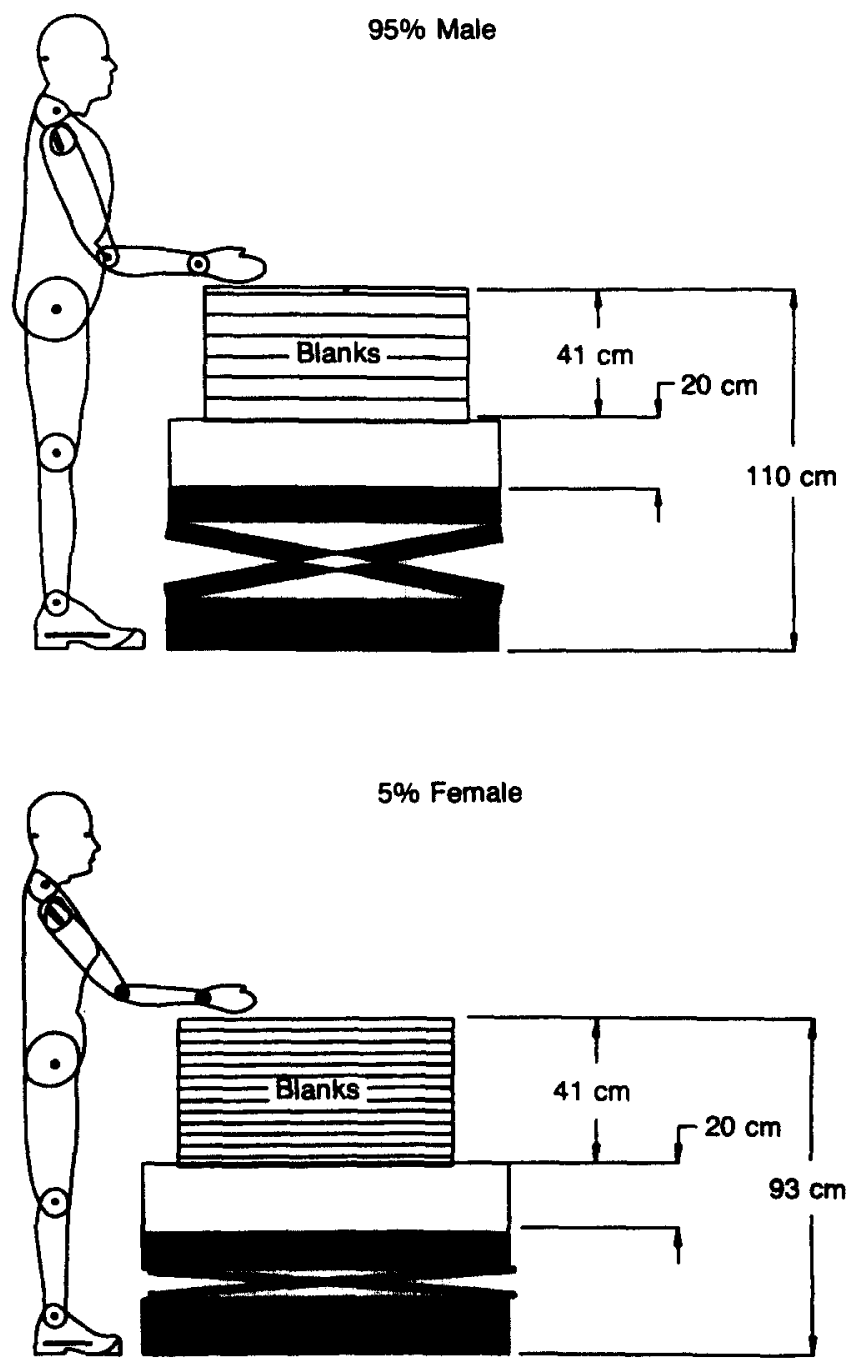

Fig. 6 Blanks on an adjustable stock table 

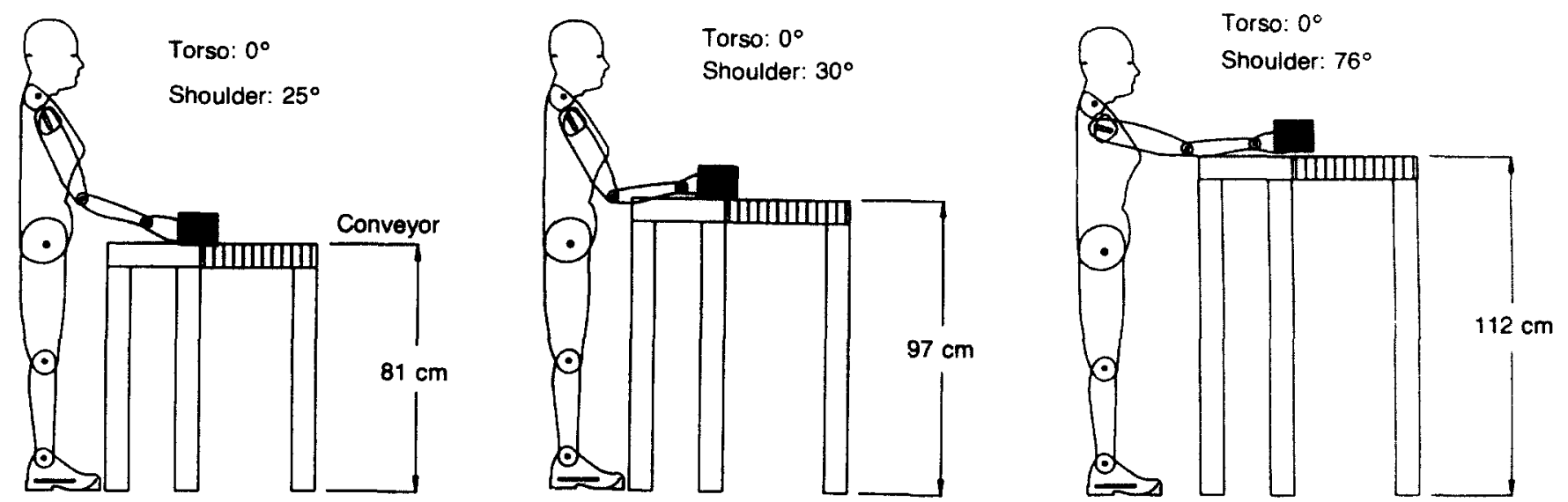

Fig. 7A Fifth percentile female lifting a load from conveyors of varying heights
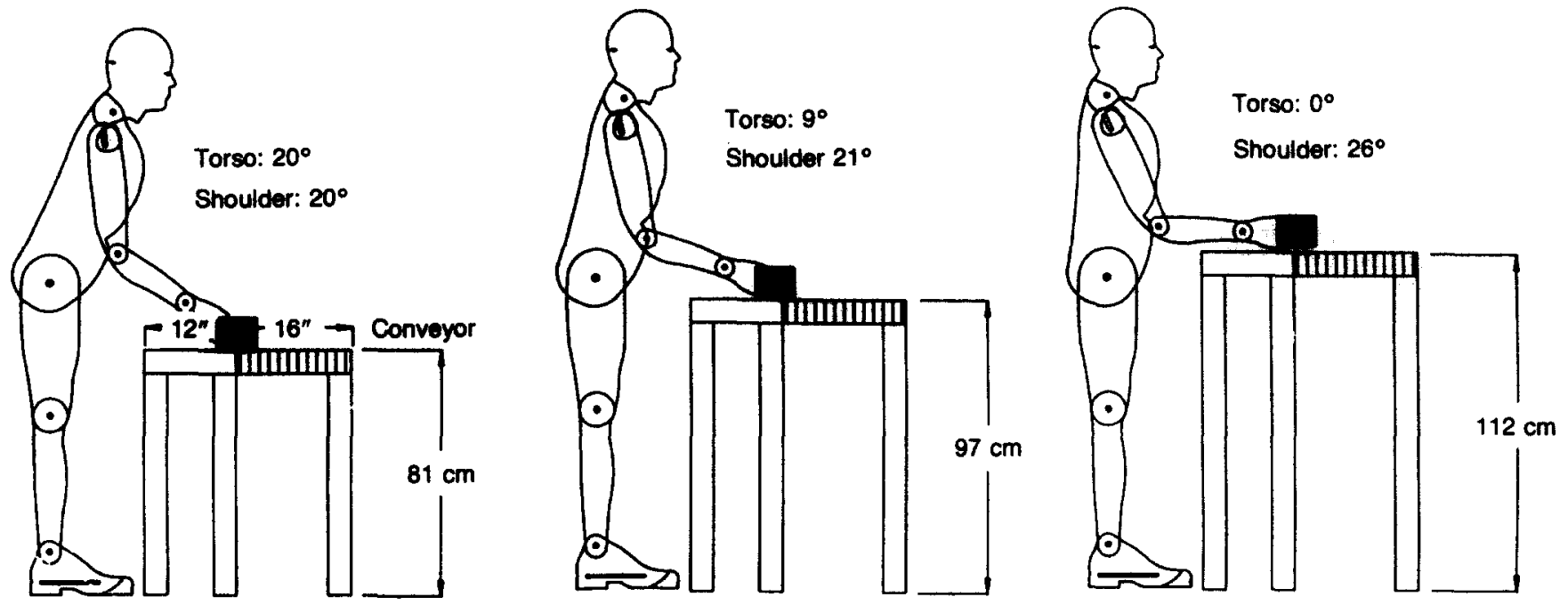

Fig. 7B Ninety-fifth percentile male lifting a load from conveyors of varying heights

population, the limitations of the design method must be taken into account. The limitations of a job analysis macro could be written out on the screen each time the macro is run to inform the user.

The manikins which have been presented in this paper are two-dimensional and the designer can view their movements only in the sagittal plane. Unfortunately, a wide variety of potential operator movements cannot be accounted for using this method. For example, it is impossible to demonstrate forearm rotation or reaching across the body. Manikins are a valuable tool to use during workstation design as long as the designer is aware of their limitations. Adjustments can be made for reaching out of the sagittal plane by reducing the length of the extremity to correspond to the planar projection.

It is not possible to predict the exact sequence of joint angles that might be preferred by a given subject, but it is possible to determine if it is likely for someone of a given size fixed at one location to reach an object positioned at another location and the range of potential postures. This method is not intended for naive users; however, people familiar with postural issues and anthropometric data should find this method a significant improvement over the use of mechanical drawing boards and postural templates.

\section{Summary}

One of the most frequently cited risk factors of upper extremity cumulative trauma disorders is stressful postures. Computer aided drafting can be used for studying the interaction between workers and workstations. Problems can be identified and solutions may be generated and evaluated to ensure that new designs remedy the problems. Twodimensional manikins were digitised and the AutoCAD software package was used for developing routines to manipulate manikins and develop parametric designs of the workstation components. The use of CAD was demonstrated to be an effective method for identifying postural stresses and redesigning the workstation to control the identified stresses. At the very minimum, it helps the analyst narrow the range of possible postures for a specific workstation.

\section{Acknowledgement}

Partial funding and support was provided by Ford Body and Assembly Operations.

\section{References}

Armstrong, T.J. 1983, An ergonomics guide to carpal tunnel syndrome. Amer Indust Hygiene Assoc, Akron, Ohio. 
Armstrong, T.J., and Chaffin, D.B. 1979, J Occup Med, 21, 481-486. Carpal tunnel syndrome and selected personal attributes.

Armstrong, T.J., and Silverstein, B.A. 1987, Clin Concepts in Regional Musculoskeletal Illness, 333-354. Upper extremity pain in the workplace - Role of usage in causality.

Armstrong, T.J., Castelli, W., Evans, F., and Diaz-Perez, R. 1984,J Occup Med, 26, 197-201. Some histological changes in carpal tunnel contents and their biomechanical implications.

Armstrong, T.J., F ine, L.J., Radwin, R.G., and Silverstein, B.S. 1987 , Scand $J$ Work Environ Health, 13, 286-289. Ergonomics and the effects of vibration in hand-intensive work.

Armstrong, T.J., Foulke, J., Joseph, B., and Goldstein, S. 1982, A mer Ind Hygiene A ssoc J, 43, 103-116. An investigation of cumulative trauma disorders in a poultry processing plant.

Armstrong, T.J., Radwin, R.G., Hansen, D.J., and Kennedy, K.W. 1986, Human Factors, 28, 325-336. Repetitive trauma disorders: Job evaluation and design.

Badler, N.I., Korein, J.D., Korein, J.U., Radack, G.M., and Brotman, L.S. 1985, The Visual Computer, 1, 212-220. Positioning and animating human figures in a task-oriented environment.

Bittner, A.C., Jr, Glenn, F.A., Harris, R.M., lavecchia, H.P., and Wherry, R.J., Jr. 1987, CADRE: A family of manikins for workstation design. Trends in ergonomics/human factors IV, 733-740.

Case, K., Porter, J.M., and Bonney, M.C. 1986, SAMMIE: A computer aided design tool for ergonomists. Proc Human Factors Soc 30th Ann Meeting; 694-698.

Evans, S.M. Some computational problems in developing computerized man-models. In: R. Easterby, K.H.E. Kroemer and D.B. Chaffin (Eds), Anthropometry and biomechanics theory and application, 103-108. Plenum, New York.

Hagberg, M. 1982, J Human Ecology, 11, 99-108. Local shoulder muscular strain: Symptoms and disorder.

Herberts, P., Kadefors, R., Andersson, G., and Petersen, I. 1981, A cta Orthop Scand, 52, 299-306. Shoulder pain in industry: an epidemilogical study on welders.

Hoffman, G. 1981, Am Fam Pract, 23, 103-110. Tendinitis and bursitis.
Kennedy, K.W. 1982, Workspace evaluation and design: USAF drawing board manikins and the development of cockpit geometry design guides. In: R. Easterby, K.H.E. Kroemer and D.B. Chaffin (Eds), Anthropometry and biomechanics theory and application, 205-213, Plenum, New York.

Keyserling, W.M. 1986, Ergonomics, 29(4), 569-583. Postural analysis of the trunk and shoulders in simulated real time.

Luconi, F.L., Malone, T.W., and Morton, M.S. 1986, Sloan Management Rev. Expert systems: The next challenge for managers.

NASA. 1978, Reference publication 1024.

Putz-Anderson, V. 1988, Prevention strategies adopted by select countries for work-related musculoskeletal disorders from repetitive trauma. Trends in ergonomics/human factors V, 601-611.

Roebuck, J.A., Jr, Kroemer, K.H.E., and Thomson, W.G. 1975, Engineering anthropometry methods. Wiley, New York.

Rothwell, P.L., and Hickey, D.T. 1986, Three-dimensional computer models of man. Proc Human Factors Soc 30th Ann Meeting, 216-220.

Rubinstein, R., Hersh, H., and Ledgard, H. 1984, The human factor. Digital Equipment Corp, New York.

Silverstein, B.A. 1985, The prevalence of upper extremity cumulative trauma disorders in industry. PhD Dissertation, Epidemiologic Science, The University of Michigan, Ann Arbor.

Sperling, L. 1980, Test program for work gloves. Research Report 1980: 18, Umeả: Department of Occupational Safety, Division for Occupational Medicine, Labor Physiology Unit, p 29.

Tichauer, E. 1966, J Occup Med, 8, 63-71. Some aspects of stress on forearm and hand in industry.

Tichauer, E. 1976, Ind Engng, 45-56. Biomechanics sustains occupational safety and health.

Williamson, D.K., Chrenko, F.A., and Hamley, E.J. 1984, Applied Ergonomics, 15(1), 25-30. A study of exposure to cold in cold stores. 